\title{
Risk, Markets, Games, and Networks
}

The 20 research articles collected in this issue share, in addition to their topical relations, one important feature: they present results obtained from research groups associated in the COST action P10 'Physics of Risk'. COST is an intergovernmental framework for 'European Cooperation in Science and Technology', allowing the coordination of research on a European level. The action P10 was funded between September 2003 and June 2008 and involved more than 80 scientists in 21 countries across Europe.

The main objective of the action was to apply methods developed in statistical physics to accomplish a new quantitative understanding of the assessment of risk. This way it departed from usual activities in the field of risk management. In fact, in recent years statistical physics has successfully contributed to many interdisciplinary fields ranging from regulatory networks in biology to financial market models. Therefore, a transfer of methods and tools from statistical physics into the area of risk assessment should be a promising endeavour.

An additional objective was to increase the understanding of the fundamental principles underlying the problems of uncertainty and complexity in the socio-economic realms. This is of great relevance to the society, in particular for economic endeavors. Objective assessment of risk and quantitative representations of information that support decisions has a vital role to play in many other areas, too.

To encompass this broader perspective, the scientific programme of the COST action P10 rested on three mutually interlocking Working Groups (WG). WG 1 'Physics and Risk' developed direct studies of phenomena in financial markets and brought together physicists with financial experts, economists and other pertinent disciplines. Observed time series and related measurements formed the basis of activity, analyzing properties at a macroscopic level. WG 2 'Agent Models' encompassed efforts to develop descriptions at the microscopic level. For example, to develop and understand economic activity, one requires a better characterisation of the nature and dynamics of the interacting agents. This also involved sociologists and economists who are concerned with social behaviour, decision processes, and game-theoretical interactions. WG 3 'Networks, Dynamics and Topology' aimed to put this research into a coherent framework rooted in the nature of the underlying networks of interacting agents. This generated new insights into the structure and dynamics of complex systems from nonlinearity, emergence and self-organized criticality.

Similar to other participating countries, Switzerland has established its own nationally-funded research projects as part of the COST-P10 activities (see http://www.sg.ethz.ch/research/projects/cost), which were supported by the State Secretariat for Education and Research (SER Project Number: C05.0039) between January 2007 and November 2008. These allowed the Swiss research institutions to actively participate in the action and to intensify their collaboration with leading European teams, but also inside Switzerland.

The results obtained in the five different Swiss projects hosted in Zurich, Geneva, Fribourg and Winterthur form the core of this topical issue. With 10 papers, half of the contributions originated from Swiss research institutions, some of these in close collaboration with other European nodes. The remaining papers were contributed by European partners of the COST action P10. They complete the overview of the broad research activities within the three working groups.

The first four papers are devoted to the problem of Systemic Risk which, different from the public opinion, is not only a problem in financial systems but also in social systems (e.g. epidemics) or in technical systems (e.g. blackout of power grids). These systems may exhibit states of collective failure, dependent on the interactions of their subunits and external conditions, which are reminiscent of phase transitions in physical systems.

Yet, the predominant interest in systemic risk today is in Financial Markets, which are investigated in more detail in the four papers of the respective section. Interestingly, despite years of intense investigations of financial systems in the field of econophysics, the dynamics of financial interactions is still not fully understood. Empirical investigations 
still detect unknown regularities and network structures in observed data, which are aimed to be captured by means of refined agent-based models.

In order to obtain a more fundamental understanding of the underlying interaction dynamics, it is necessary to consider the strategic behavior of agents. Thus, the five papers grouped into Section Games and Decisions address the role of uncertainty or time lags in decisions, but also herding effects and competition, which may enhance or prevent the emergence of ordered states in socio-economic systems.

A particular and long-standing research problem in economics and the social science is the promotion of cooperative behavior in multi-agent systems. Three papers of the current selection address the transition towards Cooperation from a physics perspective where it, again, shows some similarities to phase transitions. While individual agents may get some benefit from defecting in a strategic interaction, it is shown here that additional features such as migration or localized centers of cooperation may enhance the transition towards global cooperation, but alternating scenarios are possible as well.

Using the Complex Networks approach, interactions between agents can be described as links between nodes. In addition to the topology of these networks, which for real social systems have to be revealed from data, also the link capacity plays a considerable role, for example in information transfer. The last four papers of this topical issue address these problems from various perspectives. They provide empirical insights, but also formal models for the characterization of complex networks.

As the guest editors of this topical issue, we hope that the current selection of papers provides a good overview of the research activities within the COST action P10, both in Switzerland and Europe. The results presented should also boast future research activities within the new COST action MP0801 'Physics of Competition, Cooperation and Conflicts' which succeeded COST P10 and started in September 2008.

The Guest Editors

Frank Schweitzer, Stefano Battiston, Claudio J. Tessone

Chair of Systems Design, ETH Zürich, Switzerland 